\title{
FATORES QUE INTERFEREM NO ACESSO AOS SERVIÇOS DE SAÚDE MENTAL POR CRIANÇAS E ADOLESCENTES
}

\section{Factors that interfere in access to mental health services by children and adolescents}

\author{
João Mário Cubas (OrclD) \\ Pontifícia Universidade Católica do Paraná - PUCPR - Curitiba (PR) - Brasil \\ Dilmeire Sant'Anna Ramos Vosgerau (OrcID) \\ Pontifícia Universidade Católica do Paraná - PUCPR- Curitiba (PR) - Brasil \\ Deborah Ribeiro Carvalho (OrcID) \\ Pontifícia Universidade Católica do Paraná - PUCPR- Curitiba (PR) - Brasil
}

\section{RESUMO}

Objetivo: Identificar fatores que dificultam ou facilitam o acesso aos serviços de saúde mental por crianças e adolescentes, em situação de acolhimento institucional. Métodos: Passaram pela aplicação de três questionários com questões abertas a profissionais atuantes em CAPSi, escolas especiais e unidades de acolhimento institucional de uma capital, no Sul do Brasil. Tratam-se de pessoas que atendem crianças e/ou adolescentes com deficiência intelectual e/ou transtorno mental e acolhidas. Inicialmente, foi utilizada a técnica de Análise de Conteúdo, complementada pelos Ciclos de Codificação de Saldaña, apoiada pelo software ATLAS.ti. Os extratos de texto foram codificados/categorizados, sendo geradas redes e queries, a partir dos principais fatores levantados. Resultados: Observou-se que emergiram duas categorias: facilidades no acesso e dificuldades no acesso, sendo identificados 21 fatores relacionados a facilidades ao acesso aos serviços de saúde mental e 37 fatores relacionados a dificuldades. Aparece com maior frequência a reinserção social por meio dos serviços substitutivos como facilitador. Contudo a falta de capacitação profissional, a falta de estruturas especializadas para o atendimento desse público e a falta de recursos humanos foram as dificuldades mais acentuadas. Conclusão: Acentuar que essas técnicas contribuíram na identificação de fatores, minimizando o grau de subjetividade, constituindo uma estratégia que propicia a instrumentalização de processos de tomada de decisão.

Descritores: Pesquisa Qualitativa; Acesso aos Serviços de Saúde; Crianças.

\section{ABSTRACT}

Objective: Identify factors that hinder or facilitate access to mental health services for children and adolescents in an institutional setting. Methods: The methods used included the application of three questionnaires with open questions to professionals working in CAPSi, special schools and foster care units of a capital city in southern Brazil, that provide care to children and / or adolescents with intellectual disabilities and / or mental disorders. It was initially used the Content Analysis technique, complemented by the Coding Cycles of Saldaña, supported by the software ATLAS.ti. The text extracts were coded and categorized, generating networks and queries, based on the main factors raised. Results: The results showed that two categories emerged: access facilities and access difficulties, with 21 factors related to facilities for access to mental health services and 37 factors related to difficulties identified. Social reintegration through substitutive services as a facilitator appears more frequently. However, the lack of professional qualification, the lack of specialized structures to attend this public and the lack of human resources were the most significant difficulties. Conclusion: It should be emphasized that these techniques contributed to the identification of factors, minimizing the degree of subjectivity, constituting a strategy that facilitates the instrumentalisation of decision-making processes.

Descriptors: Qualitative Research; Health Services Accessibility; Children. 


\section{INTRODUÇÃO}

A definição do termo "acesso" ainda é permeado de diversas nuances. Giovanella e Fleury(1) discutem que o acesso é a categoria central para a análise das inter-relações entre usuários e serviços de saúde. As mesmas pesquisadoras apresentam essa noção como um conceito geral que sumariza o conjunto de dimensões específicas que descrevem a adequação entre os usuários e o sistema de cuidados em saúde. A complexidade do conceito de acesso aos serviços de saúde está relacionada com o fato de que o acesso explica o grau de ajuste entre as necessidades dos usuários e a oferta de determinado serviço de saúde.

Contudo, as barreiras de acesso resultam de uma combinação de fatores, descritos por Falavina e Cerqueira(2), tais como os seguintes: geográficos, referentes à distância até o serviço e tempo utilizado pelo usuário no deslocamento até a unidade de saúde; organizacionais, relacionados ao modo como uma unidade oferece seus serviços, além de outros, como o tempo de espera pelo atendimento médico e a facilidade ou não na realização de exames laboratoriais e clínicos; e econômicos, relativos aos custos de transporte e ao consumo de tempo demandado.

Apesar da premissa de que crianças e adolescentes são sujeitos de direitos, tendo garantida sua proteção integral, inclusive o acesso aos diversos serviços, Teixeira, Couto e Delgado(3) apontam que o acesso permanece aquém da necessidade dessa população. Vale destacar que crianças e adolescentes são um público vulnerável, prioritário para as intervenções. Ressalta-se, ainda, a ideia de que crianças e adolescentes, com deficiência ou transtorno mental, enfrentam as mais variadas violações de direitos, destacando-se a discriminação, a falta de acessibilidade nos planejamentos urbanos nas cidades, a ausência de adequação à sua presença na rede pública de ensino e a falta de suportes públicos para que as famílias possam prover o cuidado e tratamentos adequados. A falta do tratamento adequado tem sido particularmente um ponto importante para a institucionalização infantil ${ }^{(4)}$.

Em muitos casos, quando esgotados os recursos familiares, crianças e adolescentes com deficiência ficam sob a tutela do Estado e aos cuidados de terceiros. Inúmeras reflexões acerca do acolhimento institucional desse público podem ser elencadas: os motivos do acolhimento, sua deficiência (ou deficiências) e os agravos em saúde que necessitam de acompanhamento técnico. No seu caso específico, há a necessidade de uma articulação garantindo o acesso aos serviços de saúde mental, fazendo-se essencial identificar os fatores que facilitam esse acesso, como também aqueles que o dificultam.

Contudo, mesmo com acolhimento, que é uma das medidas de proteção sancionadas pelo Estatuto da Criança e do Adolescente $(E C A)^{(5)}$, muitos dos direitos de crianças e adolescentes ainda se encontram violados. A literatura aponta como principal a violação do direito fundamental da convivência familiar e comunitária( ${ }^{(6)}$. Porém, conforme dados do Relatório da Situação Mundial da Infância( ${ }^{(7)}$, em muitos países, crianças com deficiência ainda são acolhidas em instituições, que raramente oferecem o cuidado individual que esses sujeitos necessitam para que possam desenvolver plenamente suas capacidades. Muitas vezes a qualidade do ensino, dos cuidados médicos e de reabilitação que são providos pelas instituições são insuficiente ${ }^{(7)}$.

$\mathrm{Na}$ atualidade, o acolhimento de crianças e adolescentes ficou restrito a ambientes menores, diferentemente ao praticado pelas instituições totalitárias no passado. Esse reordenamento dos serviços de acolhimento propicia a vivência de uma casa, respeitando suas individualidades e evitando especificidades como, por exemplo, crianças com deficiência. A Resolução CNAS n 109/2009( ${ }^{(8)}$ distingue as novas formas de acolhimento institucional para crianças e adolescentes nas seguintes modalidades: Casa-lar e Abrigo institucional. Um dos objetivos desse novo formato é considerar a proteção do direito à convivência comunitária. Ainda, esse novo modelo de reordenamento necessita de uma articulação da rede de serviços que possa garantir o acesso desse público aos serviços de saúde mental.

No campo da saúde, há um crescente interesse pelo emprego de procedimentos qualitativos. Contudo deve-se atentar ao fato de que esse tipo de pesquisa requer um aparato teórico-metodológico que possa garantir e inferirse algo, propondo evidenciar novas ideias sobre o que até então já é conhecido( ${ }^{(9)}$. Estudos qualitativos em saúde buscam envolver a intensidade e não somente a extensão do fenômeno que está sendo estudado(10,11).

Estudos qualitativos que visem identificar esses fatores, que interferem no acesso, proporcionam maior objetividade para a constituição de programas e serviços em saúde, podendo instrumentalizar gestores no processo de tomada de decisão, na garantia de direitos de crianças e adolescentes acolhidos ${ }^{(12)}$.

Dentre as diversas técnicas de pesquisa de campo em saúde, ressalta-se a associação da técnica de Análise de Conteúdo de Bardin ${ }^{(13)}$ com os Ciclos de Codificação de Saldaña ${ }^{(14)}$, proposta por Vosgerau, Pocrifka e Simonian ${ }^{(15)}$, com vista à melhor sistematização do processo de codificação, com intenção de minimizar a subjetividade do pesquisador no processo de análise de dados qualitativo(12).

O processo de Análise de Conteúdo, segundo Bardin(13), introduzido em meados da década de 1970, iniciase com a pré-análise, que consiste na organização dos materiais que serão utilizados, existindo, nesta fase, um 
protocolo composto por quatro etapas (leitura flutuante, delimitação do que será realmente analisado, formulação de hipóteses e objetivos e elaboração de indicadores por meio de extratos de texto analisados ${ }^{(15)}$.

Para Moraes ${ }^{(16)}$, a Análise de Conteúdo compreende procedimentos especiais para o processamento de dados científicos, podendo-se considerar-se uma única técnica, mas marcada por uma grande variedade de formas e adaptável a um campo de aplicação muito vasto, seja qual for a forma de comunicação. É necessário atentar para o fato de que, embora o consenso ou o acordo intersubjetivo sobre o que significa uma mensagem simplifique a Análise de Conteúdo, essa coincidência de significados não é indispensável. Os valores culturais e a linguagem natural do entrevistado e do pesquisador, bem como a linguagem cultural e seus significados, exercem certa influência nos dados, não podendo ser ignorados pelo pesquisador. Sendo assim, a Análise de Conteúdo é uma interpretação pessoal do pesquisador com relação à percepção que tem dos dados.

Já Saldaña ${ }^{(14)}$ apresenta sua proposta de análise de dados qualitativos por meio de um processo de codificação sistemático e cíclico. O autor propõe a elaboração de dois ciclos de codificação dos dados, com 31 possibilidades de elaboração desses códigos. Na primeira etapa ou primeiro ciclo de codificação, explana a necessidade de que o pesquisador proceda à leitura inicial dos dados, realizando suas primeiras interpretações por memos ou memórias. Nessa fase, as informações importantes devem ser demarcadas. O segundo ciclo pode ou não ocorrer ${ }^{(15)}$, como também voltar-se ao primeiro ciclo, se entendido necessário para o aprofundamento da análise.

Para otimizar este processo de análise, a utilização de software para a análise de dados qualitativos, como o ATLAS.ti, vem sendo amplamente discutida e implementada.

Segundo Queiroz e Cavalcante ${ }^{(17)}$, o uso do ATLAS.ti é indicado para análise longitudinal, em que se usam instrumentos diversos e complementares, com o principal objetivo de auxiliar o pesquisador a organizar, registrar e acompanhar os registros efetuados, o que contribui com a confiabilidade da pesquisa. Com o software, é possível analisar e gerenciar diversos tipos de documentos ou instrumentos de coleta de dados, tais como os seguintes: respostas a questões abertas de questionários; relatórios de observação; cartas; registros áudio para transcrição de entrevistas não estruturadas, músicas, reuniões e palestras; imagens, podendo ser fotos, desenhos, pinturas e outros formatos; e vídeos, incluindo gravação de reportagens, aulas, filmes, entre outros trabalhos. Além dessas possibilidades, o ATLAS.ti oferece ferramentas que podem facilitar e aprimorar a aplicação da técnica de Análise de Conteúdo(15).

Por sua vez, para Pocrifka ${ }^{(18)}$, sua utilização permite uma organização contínua na base de dados, o que proporciona, em termos de ferramentas, mecanismos de procura rápida e flexível, possibilitando estabelecer palavras-chave ou "rótulos/etiquetas" em segmentos do texto, com vistas a encontrar automaticamente ou recuperar posteriormente um documento. O software também permite atrelar segmentos de dados uns aos outros, estabelecendo categorias, teias ou redes de informação para utilização em curto, médio e longo prazo, além de possibilitar ao pesquisador a criação de diagramas que facilitam a visualização dos resultados. Ainda é de acentuar que beneficia o campo da investigação social, pois admite a interação entre teoria e prática e um retorno fácil a etapas de codificação, quando se percebe a necessidade de rever possíveis vieses da codificação.

Vosgerau et al. ${ }^{(15)}$ propõem, em seu estudo, as seguintes etapas para a análise de dados qualitativos associando as técnicas de Bardin ${ }^{(3)}$ e Saldaña ${ }^{(14):}$ leitura flutuante; seleção das quotations (citações); criação de memos (memórias) analíticos; e codificação (em um ou dois ciclos), aliando a esses procedimentos a utilização do software ATLAS.ti.

Objetivando confrontar o conjunto de fatores já identificados na literatura ${ }^{(19)}$, esta pesquisa buscou levantar, a partir de pesquisa de campo, os fatores associados ao acesso aos serviços de saúde mental, por crianças e adolescentes com deficiência intelectual e/ou transtorno mental, em situação de acolhimento, em uma capital no sul do Brasil. Neste artigo, é evidenciado o uso da técnica de Análise de Conteúdo ${ }^{(13)}$, associada aos Ciclos de Codificação(14), conforme sugerido por Vosgerau et al. ${ }^{(15)}$.

\section{MÉTODOS}

Neste estudo de campo, evidencia-se o processo de análise qualitativa de um projeto mais amplo, tendo como cenário de pesquisa as unidades de acolhimento, nas modalidades Casa-lar e Abrigo institucional, que atendem crianças e/ou adolescentes com deficiência intelectual e/ou transtorno mental associado; os Centros de Atenção Psicossocial Infantil (CAPSis) (serviços substitutivos aos hospitais psiquiátricos, por meio da Reforma Psiquiátrica(20,21), que visam à reinserção social do indivíduo no território, evitando a segregação) e escolas especiais de uma grande mantenedora em uma capital do Sul do Brasil. A seleção deste cenário decorre do reordenamento dos serviços de acolhimento ${ }^{(8)}$, que propõe uma ação intersetorial de proteção a essa população, sendo importante verificar como se dava o acesso aos serviços de saúde mental, bem como a respectiva disponibilidade frente à demanda. 
Participaram do estudo profissionais que atuavam nos respectivos serviços. Não houve delineamento por profissões, sendo selecionados todos os profissionais que prestavam atendimento direto a esta população.

As informações foram coletadas por meio de três questionários, formulados pelos autores, contendo treze questões abertas que cobriam três temáticas: a) profissionais (se a reforma psiquiátrica trouxe avanços no atendimento de crianças e adolescentes; se os atendimentos eram priorizados para essa população; se existia dificuldade no ingresso ou permanência nos serviços; os desdobramentos frente ao diagnostico dual (deficiência intelectual e transtorno mental); se o reordenamento dos serviços de acolhimento contribuiu para o acesso; se os equipamentos de saúde encontram-se preparados para o atendimento da clientela acolhida); b) crianças e adolescentes acolhidos (se há prioridade no acesso; se existe dificuldade de permanência; se os serviços de saúde conseguem contemplar os atendimentos que a criança ou adolescente demanda); e c) unidades de acolhimento (se o acesso era priorizado para essa população; se o reordenamento dos serviços de acolhimento contribuiu para o acesso; quais seriam os elementos que facilitam ou dificultam o acesso aos serviços de saúde mental).

Os textos foram transcritos independentemente do questionário de origem para planilhas eletrônicas e, após parametrizações, foram tratados qualitativamente por meio do software ATLAS.ti, versão 7.5.10.

Foram entrevistados 64 profissionais participantes e oito coordenadores de Unidades de Acolhimento Institucional (UAIs), totalizando 118 documentos (64 profissionais, 46 crianças e adolescentes acolhidos e oito Unidades de Acolhimento) apropriados para a análise.

A coleta de dados, em campo aberto e com questões abertas, se justifica dada a rotina de trabalho dos profissionais envolvidos, frente a sua disponibilidade. O olhar sob as três perspectivas propostas, profissional, instituição e criança e adolescente permite analisar possíveis fatores, sem prejuízo de um viés a partir de um único foco, criando assim uma triangulação metodológica para a análise qualitativa.

Primeiramente, foi realizado um survey (levantamento) individual das três planilhas (uma de cada questionário), em unidades hermenêuticas (projetos) diferentes no ATLAS.ti, posteriormente unificadas em um único projeto, utilizando o recurso Merge, para junção de projetos, disponível no software. Antes de iniciar o processo de análise, os documentos foram agrupados em famílias por tipo de questionário ( $A, B$ e $C$ ), tipo de equipamento (UAls, CAPSis e escolas especiais) e profissionais - psicólogos, visando ao aprofundamento da análise, identificando no fim as respostas, por meio dos filtros do ATLAS.ti.

Conforme foi referido por Vosgerau et al. ${ }^{(15)}$, este estudo foi desenvolvido buscando associar a Análise de Conteúdo de Bardin ${ }^{(13)}$ aos Ciclos de Codificação elencados por Saldaña ${ }^{(14)}$, obedecendo às seguintes etapas propostas de análise de dados qualitativos: 1) leitura flutuante; 2) seleção das quotations; 3) criação de memos teóricas; 4) codificação em primeiro, segundo e terceiro ciclo (refinamento); 5) criação de famílias de códigos (para auxiliar na quantificação dos códigos); e 6) criação de redes e queries (relatórios).

A seleção das quotations e sua posterior codificação em primeiro ciclo possibilitaram a aproximação dos pesquisadores com o material preparado para a análise. Nessa seleção, foi priorizada a identificação preliminar dos fatores objeto deste estudo. Já em segundo ciclo, os códigos, além de sofrerem refinamento, eliminando-se os redundantes, foram confrontados com os fatores identificados previamente na literatura, a partir das bases de dados - Literatura Latino-Americana e do Caribe, em Ciências da Saúde (Lilacs), Scientific Electronic Library Online (SciELO), como também da biblioteca digital da Plataforma da Coordenação de Aperfeiçoamento de Pessoal de Nível Superior (Capes), com os seguintes descritores: acesso em saúde mental de crianças, acesso em saúde mental de adolescentes e acesso em saúde mental infantil ${ }^{(19)}$. No terceiro ciclo, o estabelecimento de categorias ${ }^{(13)}$ permitiu a classificação de elementos por diferenciação, neste caso, a partir das dimensões do acesso em saúde propostas por Giovanella e Fleury ${ }^{(1)}$, a saber: política, econômico-social, técnica e simbólica. Dito de outro modo, a ordenação dos códigos (fatores) foi efetuada por meio de uma estrutura conceitual do acesso. Devido ao volume de informação qualitativa, nesta etapa foi fundamental o uso dos recursos estatísticos oferecidos pelo software para selecionar as categorias relevantes e, a partir delas, construir as famílias e retornar as informações qualitativas essenciais para a construção das redes que explicavam os fatores levantados. Destaca-se neste lugar que o uso de ferramentas estatísticas combinadas a análise qualitativa são importantes recursos, quando se trabalha com grande volume de informações qualitativas, pois permite que o pesquisador localize as informações que são mais relevantes para a compreensão do fenômeno a ser estudado(22,23).

Como recursos estatísticos foram consideradas neste estudo apenas as frequências relativa e absoluta, no sentido de quantificar os achados.

Vale destacar que a criação de famílias de códigos possibilita a classificação dos elementos por diferenciação, oportunizando o reagrupamento a partir dos critérios predefinidos ${ }^{(24)}$. Neste estudo, o critério para agrupamento era distinguir os fatores relacionados ao avanço e às dificuldades no acesso aos serviços de saúde mental. 
A pesquisa foi aprovada pelo Comitê de Ética em Pesquisa de uma universidade, após submissão do projeto via Plataforma Brasil, mediante o Parecer $n^{\circ}$ 1.194.564.

\section{RESULTADOS E DISCUSSÃO}

A partir da metodologia proposta, foram identificados 396 extratos de texto. Num primeiro ciclo de análise, esses extratos foram codificados em 118 códigos. O segundo ciclo de codificação possibilitou uma redução para 58 códigos, os quais foram subdivididos nas quatro dimensões de acesso propostas por Giovanella e Fleury ${ }^{(1)}$, propiciando um terceiro ciclo de codificação.

Os 58 códigos (ou subcategorias) foram agrupados em duas famílias de códigos: avanços no acesso e dificuldades no acesso, conforme apresentadas nas Tabelas I e II respectivamente, objetivando diferenciar os fatores relacionados ao acesso aos serviços de saúde mental.

Neste ponto, a revisão de literatura previamente apontada por Cubas ${ }^{(19)}$ contribuiu para a construção de uma matriz de análise, verificando o que os estudos publicados já traziam como fatores relacionados ao acesso aos serviços de saúde mental, correlacionando com os que seriam identificados neste trabalho.

Com relação aos avanços, os extratos foram subcategorizados em 21 fatores (ou códigos), que podem ser visualizados na Tabela I.

Por mais que os participantes relatassem em seus discursos não haver dificuldades, os resultados da família "dificuldades no acesso" apontam que existem diversas situações onde o acesso é reprimido (Tabela II).

A reinserção social foi apontada como o principal fator de avanço no acesso, aparecendo em 17 extratos de texto. Especificamente, as famílias de documentos propostas identificaram que a reinserção social $(17=100,0 \%)$ foi citada dez vezes $(58,8 \%)$ por profissionais dos CAPSis, três $(17,6 \%)$ por profissionais das escolas especiais, três $(17,6 \%)$ por profissionais que trabalham em Unidades de Acolhimento, na modalidade Abrigo institucional e uma $(5,9 \%)$ por profissionais das Unidades de Acolhimento, na modalidade Casa-lar. Entre os profissionais psicólogos, foi citada cinco vezes nos seus extratos de texto. Salienta-se que na revisão de literatura desenvolvida por Cubas ${ }^{(19)}$ esse avanço não foi identificado.

A reinserção social pode ser entendida por meio da presença e expansão dos CAPSi, que podem facilitar o processo de inclusão social de crianças e adolescente, pois buscam a construção de uma rede local de cuidados ${ }^{(25)}$.

Tabela I - Frequência absoluta e relativa - Fatores relacionados aos avanços no acesso.

\begin{tabular}{lcc}
\hline Fatores & FA & FR \\
\hline Sem dificuldades & 67 & 35.6 \\
Reinserção social & 17 & 9.0 \\
Prioridade igualitária & 17 & 9.0 \\
Acolhimento garantia atendimento & 15 & 8.0 \\
Facilidade acesso enquanto crianças & 13 & 6.9 \\
ECA e Reforma Psiquiátrica avanços & 11 & 5.9 \\
Rede facilita acesso & 9 & 4.8 \\
Sujeitos de direito & 6 & 3.2 \\
Medida de proteção como facilitador & 5 & 2.7 \\
Reinserção familiar & 4 & 2.1 \\
CAPS possibilita novos caminhos & 4 & 2.1 \\
Atendimento humanizado & 3 & 1.6 \\
Quebra estigma & 3 & 1.6 \\
Transtorno estabilizado & 3 & 1.6 \\
Importância tratamento & 3 & 1.6 \\
Atendimento território & 2 & 1.1 \\
Comunicação profissional & 2 & 1.1 \\
Deficiência intelectual prioridade acesso & 1 & 0.5 \\
Resultados pesquisados avaliados & 1 & 0.5 \\
Ser órgão público & 1 & 0.5 \\
Profissionais em mudança & 1 & 0.5 \\
\hline
\end{tabular}

$F A=$ frequência absoluta. $F R=$ frequência relativa 
No caso do acolhimento e garantia de atendimento, esta pesquisa divergiu dos estudos da revisão(19), que apontaram a necessidade de uma melhor articulação com a rede de proteção o que pode fazer supor que crianças e adolescentes que não se encontram em acolhimento podem ter dificuldades de acesso. A medida de proteção de acolhimento garante o acesso desses sujeitos acolhidos aos serviços, atrelada ao fato de ser um público respaldado pela legislação de defesa de direitos, garantindo a prioridade absoluta como preconizada pelo ECA ${ }^{(5)}$.

Os serviços substitutivos de saúde mental, como os CAPSis, segundo os participantes, garantem novas possibilidades de tratamento, divergindo da segregação social proposta pelos antigos hospitais psiquiátricos. Contudo para a efetivação dos CAPSi evidenciam-se desafios funcionais e geram novas necessidades (como por exemplo o atendimento dessa demanda acolhida), necessitando de uma constante avaliação de suas atividades propostas em face às dificuldades comuns do Poder Público ${ }^{(25)}$.

Com relação às dificuldades, os extratos de texto foram subcategorizados em 37 fatores (códigos), sendo apresentadas por meio da Tabela II.

Tabela II - Frequência absoluta e relativa - Fatores relacionados as dificuldades no acesso.

\begin{tabular}{|c|c|c|}
\hline Fatores & FA & FR \\
\hline Falta capacitação & 63 & 18.1 \\
\hline Falta estrutura e estrutura especializada para atendimento de crianças e adolescentes & 35 & 10.0 \\
\hline Não há prioridade acesso & 24 & 6.9 \\
\hline Falta recursos humanos & 24 & 6.9 \\
\hline Adolescente pouco colaborativo & 20 & 5.7 \\
\hline Amplitude atendimento & 19 & 5.4 \\
\hline Faltam vagas ambulatório internação & 18 & 5.2 \\
\hline Falta parceria outros serviços necessitam melhorias demora & 17 & 4.9 \\
\hline Atendimento CAPS limitado & 12 & 3.4 \\
\hline Sistema não consegue garantia prot. integral rede local & 12 & 3.4 \\
\hline Falta atendimento especializado psicoterápico & 11 & 3.2 \\
\hline Perfil ingresso & 11 & 3.2 \\
\hline Pouco investimento Saúde Mental infantil poucos CAPSI & 8 & 2.3 \\
\hline Dificuldade permanência & 7 & 2.0 \\
\hline Atendimento sem atrativo - baixa adesão & 7 & 2.0 \\
\hline Falta mobilidade acessibilidade & 7 & 2.0 \\
\hline Falta recurso familiar & 5 & 1.4 \\
\hline Medicações ultrapassadas excesso ou falta & 4 & 1.1 \\
\hline Rejeição outros adolescentes no equipamento & 4 & 1.1 \\
\hline Não garante proteção integral & 4 & 1.1 \\
\hline Público vulnerável & 4 & 1.1 \\
\hline Aumento demanda & 4 & 1.1 \\
\hline Desconhecimento & 4 & 1.1 \\
\hline Atenção básica não propicia atenção no território & 3 & 0.9 \\
\hline Dificuldade elaboração projetos individuais casa & 3 & 0.9 \\
\hline Dificuldade diferenciação entre Deficiência Intelectual e Transtorno Mental & 2 & 0.6 \\
\hline Prioridade em crises somente & 2 & 0.6 \\
\hline Atendimento rede privada & 2 & 0.6 \\
\hline Locais acolhimento - falta estrutura & 2 & 0.6 \\
\hline Resistência profissional & 2 & 0.6 \\
\hline Caso grave remete instituições totais & 2 & 0.6 \\
\hline Def. intelectual dificulta acesso & 2 & 0.6 \\
\hline Dificuldade na comorbidade uso substâncias & 1 & 0.3 \\
\hline Esforços equipe manter atendimento & 1 & 0.3 \\
\hline Falta participação usuários & 1 & 0.3 \\
\hline Regionalização compromete vinculo & 1 & 0.3 \\
\hline Patologização para o acesso & 1 & 0.3 \\
\hline
\end{tabular}

$F A=$ frequência absoluta. $F R=$ frequência relativa 
Como principal fator relacionado à dificuldade no acesso aos serviços, a falta de capacitação foi a mais evidenciada, sendo verificada em 63 extratos de texto. Na Figura 2, apresenta-se a rede (network) acerca desse fator, com alguns extratos de texto das respostas dos participantes. Nessa rede, o número 63 está associado à quantidade de citações e o número 1 à categoria de análise do acesso, a que o código encontra-se relacionado (técnica). A falta de capacitação $(63=100,0 \%)$, foi citada 16 vezes $(25,4 \%)$ por profissionais dos CAPSis, dez $(15,9 \%)$ por profissionais das escolas especiais e $28(44,4 \%)$ por profissionais que trabalham em Unidades de Acolhimento, na modalidade Abrigo institucional. Esse mesmo fator apareceu seis vezes $(9,5 \%)$ nos extratos de texto contidos nos questionários acerca da criança e/ou adolescente (questionário B) e três $(4,8 \%)$ nos extratos de texto dos dirigentes nos questionários acerca da Unidade de Acolhimento (questionário $\mathrm{C}$ ). Entre os profissionais psicólogos, foi citado 13 vezes em seus extratos de texto. Ressalta-se que essa também foi a principal dificuldade apontada nos estudos que fizeram parte da revisão de Cubas ${ }^{(19)}$. Na Figura 1 é possível visualizar a continuidade dos discursos por meio das relações que o software dispõe, garantindo uma sequência entre os mesmos.

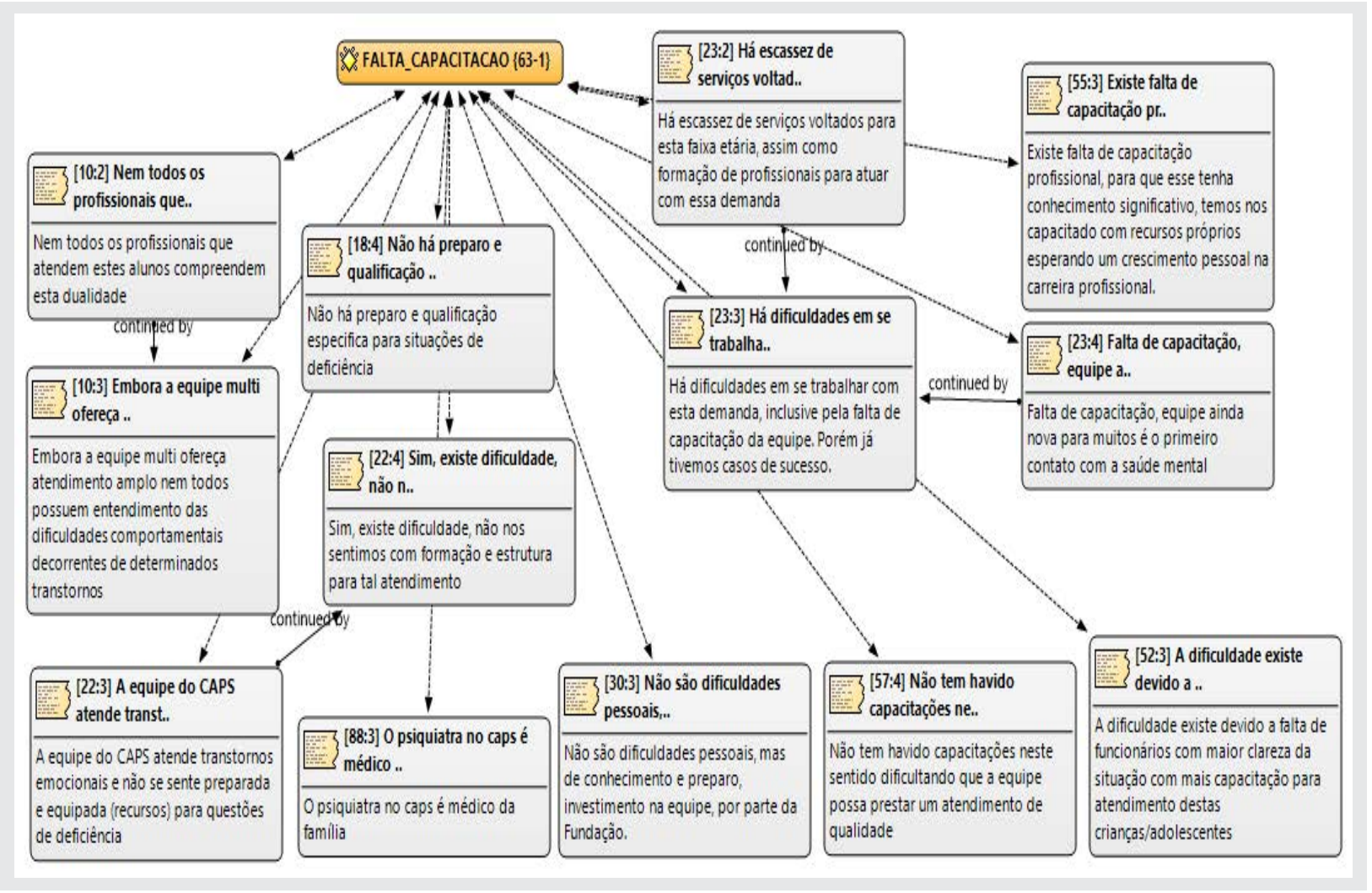

Figura 1 - Rede do código "falta de capacitação".

A falta de articulação nos serviços no território foi evidenciada nesta pesquisa, sendo um tema explorado em outros estudos e que necessita ser discutido ${ }^{(26)}$. A intersetorialidade dos serviços deve contemplar a singularidade de cada território e os cuidados em rede devem estar em funcionamento compartilhado ${ }^{(25,27)}$.

As dificuldades quanto ao diagnóstico precoce foram vislumbradas na revisão(19). Contudo os participantes não elencaram a falta de um diagnóstico como limitante no acesso, como apontado na literatura ${ }^{(28)}$. Nesse sentido, a capacitação profissional pode reduzir essa dificuldade de diagnóstico, proporcionando melhores condições de tratamento para este público ${ }^{(29)}$.

A falta de investimentos em saúde mental infantil, bem como a falta de prioridade nos serviços de saúde mental na infância se evidenciaram neste estudo, corroborando com a literatura, onde cita-se que os recursos são distribuídos de forma desigual em todo o mundo ${ }^{(28,30)}$. Atrelada a falta de investimentos, encontra-se a falta de estruturas especializadas no atendimento de crianças e adolescentes. Neste estudo, os profissionais respondentes afirmaram um número de CAPSi insuficientes ${ }^{(27)}$ e as poucas vagas para ambulatório e internação.

A falta de recursos humanos na internação também é evidenciada, por exemplo quando é referida a quantidade reduzida de psiquiatras infantis ${ }^{(26,28)}$, o que infringe a legislação que faz a menção a capacitação e educação continuada ${ }^{(21,28-30)}$.

Como dificuldades identificadas nesta pesquisa, a amplitude no atendimento entra em pauta quando se coloca em debate o sujeito com diagnóstico dual (deficiência intelectual associada a transtorno mental). A esse respeito, a 
falta de oferta de serviços de saúde mental para portadores de deficiência intelectual ${ }^{(31,32)}$ é acentuada pela ausência de capacitação profissional no atendimento desse público, sendo uma interferência significativa no acesso aos serviços, pois existe dificuldade de entendimento por parte dos profissionais de saúde mental ${ }^{(32,33)}$. Ainda no tocante à amplitude de atendimento, faz-se necessário considerar as situações de vulnerabilidade a que esses sujeitos (crianças e adolescentes acolhidos) foram expostos, demandando maior atenção e cuidado. Nesse sentido, o perfil de ingresso dessa criança ou adolescente limita, por vezes, o atendimento no serviço ${ }^{(33)}$. $O$ atendimento do CAPSi, segundo os participantes, é considerado limitado, entendendo-se que não há atrativos para a permanência de crianças e adolescentes nos serviços. A falta de atrativos para o atendimento pode evidenciar a ausência de um atendimento especializado para esse público ${ }^{(34)}$. Ressalta-se, por exemplo, a escassez de atendimento psicológico individual, tendo em vista que os atendimentos em CAPSi, em sua maioria, são coletivos, não sendo atrativos para o adolescente, agravado por sua situação de vulnerabilidade ${ }^{(26,34)}$. No caso de crianças e adolescentes com mobilidade reduzida, eles ainda sofrem pela falta de mobilidade e de acessibilidade dos equipamentos, dificultando o acesso e, também, a permanência para a continuidade do tratamento.

A criação de redes e os relatórios obtidos por meio do ATLAS.ti possibilitaram identificar associações entre as quotations, buscando a verificação de continuidade nos discursos (Figura 2), como também entre os códigos, verificando quais eram resultado ou mesmo consequência (Figura 3). Na Figura 3, identificam-se as relações de causa e efeito do código "falta de capacitação", considerando os demais códigos da família "dificuldades no acesso". Assim, verifica-se que a falta de capacitação profissional faz parte da falta de estrutura especializada no atendimento de crianças e adolescentes, bem como está relacionada à limitação no atendimento dos CAPSis, inferindo-se que existe falta de capacitação por parte da equipe.

Além de todo o processo de organização dos documentos (leitura, codificação, memos, etc.), portanto, o software possibilita a demonstração gráfica dos códigos, denominadas network, nas quais o próprio pesquisador pode inferir sobre o tipo de relação que existe entre os códigos, como exemplificado na Figura 2.

Os ciclos de codificação possibilitaram a busca pelo que era realmente essencial nos dados coletados. O processo de codificação de Saldaña ${ }^{(14)}$ complementa a proposta de $\operatorname{Bardin}^{(13)}$, uma vez que permite um detalhamento minucioso da etapa de codificação de dados, sendo capaz de identificar com precisão (diminuindo a subjetividade) a essência dos extratos de texto. A abordagem qualitativa nesse sentido, permitiu inferências a partir dos elementos que foram codificados e categorizados ${ }^{(35)}$, conseguindo-se determinar com clareza os fatores facilitadores e dificultadores no acesso e que não haviam sido encontrados na revisão de literatura ${ }^{(19)}$. A partir desse ponto, a discussão com as memos teóricas ${ }^{(14)}$ puderam vislumbrar os avanços que a legislação trouxe para esse público ou as necessidades de intervenção.

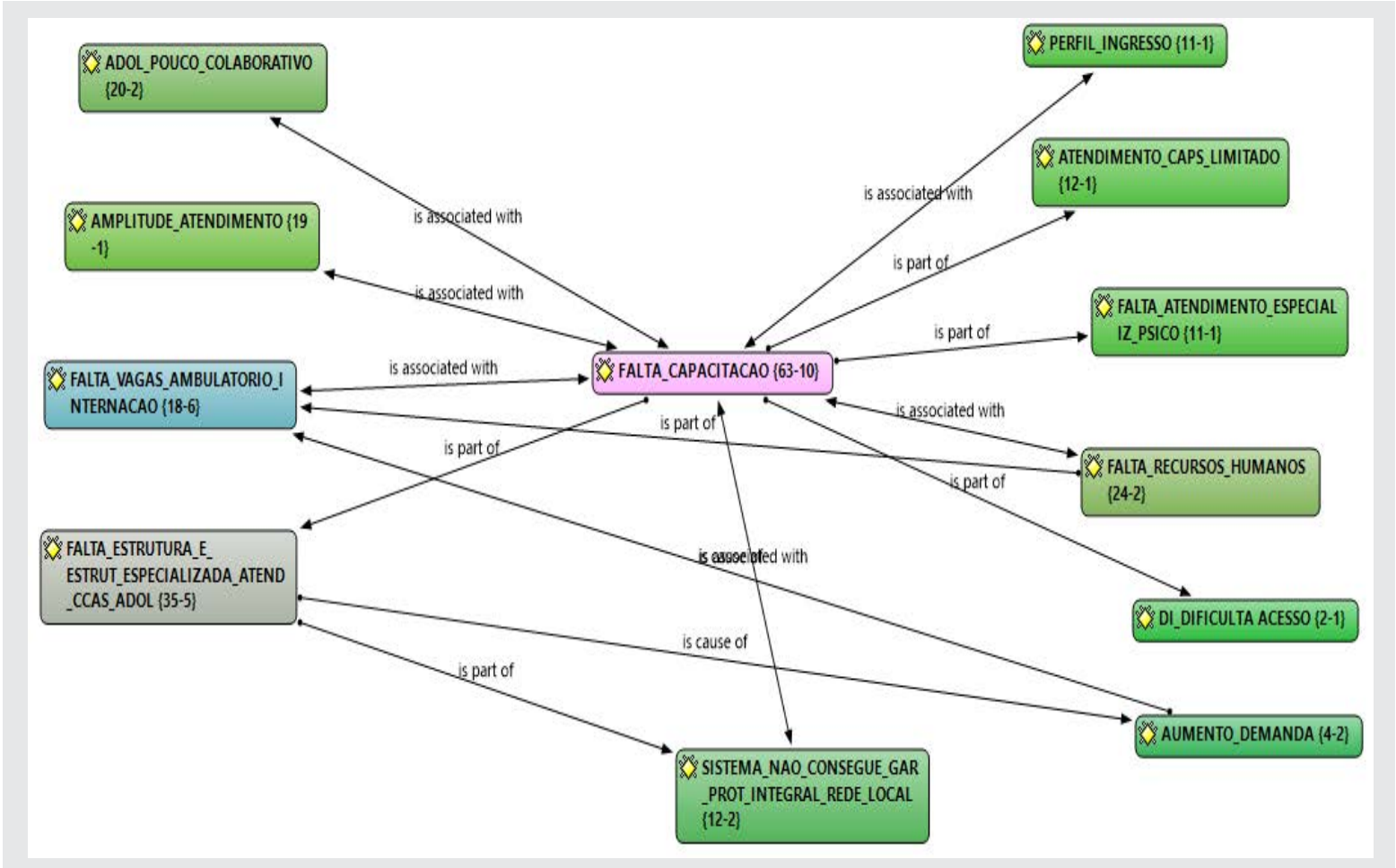

Figura 2 - Rede semântica do código "falta de capacitação" e associações com outros códigos da família "dificuldades no acesso". 
Sintetiza-se que o sistema atual de saúde mental na Federação ainda necessita de intervenções e de cuidados para a garantia do acesso aos serviços, sendo considerado como insuficiente para atender as demandas deste público estudado ${ }^{(36)}$. Há uma fragmentação dos recursos que deveriam ser dedicados a estes serviços ${ }^{(31)}$. Contudo nota-se que tanto essa fragmentação como a falta de prioridade na agenda pública não consistem em um problema exclusivo do Brasil(29-31,37). $\mathrm{O}$ acesso mais rápido e eficaz aos serviços de saúde mental, além de garantir melhores resultados para crianças e adolescentes, ainda reduziria os custos com intervenções no futuro( ${ }^{(37)}$.

\section{CONSIDERAÇÕES FINAIS}

A análise qualitativa, associando a técnica de Análise de Conteúdo de Bardin ${ }^{(13)} \mathrm{com}$ os Ciclos de Codificação de Saldaña ${ }^{(14)}$, como proposto por Vosgerau et al. ${ }^{(15)}$, possibilitou identificar fatores complementares àqueles já apontados na literatura, como também corroborou com a mesma. Por sua vez, a utilização do ATLAS.ti, aliada às etapas metodológicas propostas, oportunizou maior precisão na apropriação dos discursos, enriquecendo a análise de pesquisas de campo, na área da psicologia. A utilização do software de análise qualitativa permitiu ao pesquisador uma maior sistematização dos documentos apropriados para análise, como também de seus dados, uma vez que se disponibilizaram diversos recursos para a apreciação qualitativa. Possuem-se ferramentas de apoio estatístico, como é o caso do ATLAS.ti, possibilitando a recuperação e a discussão de informações que respondessem ao nosso problema de pesquisa.

Tanto a revisão de literatura(19) quanto esta pesquisa verificaram que a falta de capacitação profissional e de estruturas especializadas no atendimento infantil ainda são alguns dos fatores que mais inviabilizam a busca e a continuidade do tratamento por crianças e adolescentes acolhidos.

As categorias levantadas pela Análise de Conteúdo, por si só, não trazem o significado ao pesquisador, cabendo a ele interpretar e discutir esses resultados ${ }^{(38)}$. Desta forma, a metodologia proposta foi inovadora, pois identificou com detalhes novos fatores por meio da escuta dos participantes da pesquisa, tais como os seguintes códigos: 0 acolhimento, a garantia de atendimento e a dificuldade de atendimento frente à amplitude causada pelo diagnóstico dual, corrobora significativamente a importância da análise qualitativa em pesquisas em saúde pública. Donde, possibilitar a inferência sobre achados, discutindo-os. Ademais, métodos qualitativos dotados de estratégias e recursos, como os empregados neste estudo, podem instrumentalizar os governantes no processo de tomada de decisão na garantia de direitos desse público.

\section{CONFLITOS DE INTERESSE}

Os autores declaram não haver conflito de interesse, incluindo interesses financeiros específicos, de relacionamentos e afiliações relevantes ao tema, ou sobre materiais discutidos no manuscrito.

\section{AGRADECIMENTOS}

Autores agradecem à Coordenação de Aperfeiçoamento de Pessoal de Nível Superior (CAPES).

\section{FONTE FINANCIADORA:}

Coordenação de Aperfeiçoamento de Pessoal de Nível Superior (CAPES.)

Pesquisa advinda da dissertação de mestrado: Acesso aos serviços de saúde mental por crianças e adolescentes com deficiência intelectual e/ou transtorno mental em situação de acolhimento. Pontifícia Universidade Católica do Paraná, Curitiba, 2016. (Dissertação de Mestrado). 211 páginas.

\section{REFERÊNCIAS}

1. Giovanella L, Fleury S. Universalidade da atenção à saúde: acesso como categoria de análise. In Política de saúde: o público e o privado. Rio de Janeiro: Fiocruz; 1996. p. 177-198.

2. Falavina OP, Cerqueira MB. Saúde mental infanto-juvenil: usuários e suas trajetórias de acesso aos serviços de saúde. Espaç. Saúde. 2008;10(1):34-46. 
3. Teixeira MR, Couto MCV, Delgado PGG. Repercussões do processo de reestruturação dos serviços de saúde mental para crianças e adolescentes na cidade de Campinas, São Paulo (2006-2011). Estud. Psicol. 2015;32(4):695-703.

4. Vannuchi PT, Oliveira CS. Direitos humanos de crianças e adolescentes: 20 anos do Estatuto. Brasília: Secretaria de Direitos Humanos; 2010.

5. BRASIL. Lei no 8.069, de 13 de julho 1990. Dispõe sobre o Estatuto da Criança e do Adolescente e dá outras providências. Diário Oficial da República Federativa do Brasil. 1999.

6. Leite ADS. Entre o discurso e a prática: um estudo sobre a garantia do direito à convivência familiar de crianças e adolescentes com deficiência mental e/ou transtorno mental. (Dissertação de Mestrado). Rio de Janeiro: Pontifícia Universidade Católica do Rio de Janeiro; 2011.

7. UNICEF: United Nations Children's Fund. Situação Mundial da Infância: crianças com deficiência. Relatório. New York: United Nations; 2013.

8. Brasil. Ministério do Desenvolvimento Social e Combate à Fome (MDS). Conselho Nacional de Assistência Social (CNAS). Aprova a Tipificação Nacional dos Serviços Socioassistenciais, através de sua Resolução 109/2009. Diário Oficial da República Federativa do Brasil. Brasília. 2009.

9. Gonçalves H, Menasche R. Pesquisando na interface: problemas e desafios a partir da pesquisa qualitativa em saúde. Comun Saúde, Educ. 2014;18(50):449-56.

10. Araújo L, Dolina J, Petean E, Musquim C, Bellato R, Lucietto G. Diário de pesquisa e suas potencialidades na pesquisa qualitativa em saúde. Rev Bras Pesqui em Saúde. 2013;15(3):53-61.

11. Knauth DR, Leal AF. A expansão das Ciências Sociais na Saúde Coletiva: usos e abusos da pesquisa qualitativa. Comun Saúde, Educ. 2014;18(50):457-67.

12. Cubas JM, Carvalho DR, Vosgerau, DSAR. Análise de conteúdo para identificar os fatores que interferem no acesso aos serviços de saúde mental por crianças e adolescentes. Anais do $6^{\circ}$ Congresso lbero-Americano em Investigação Qualitativa (CIAIQ2017) e do 2st International Symposium on Qualitative Research (ISQR2017), 2017, Salamanca. v. 2 (2017): Atas - Investigação Qualitativa em Saúde. Salamanca. p. 285-294 . v. 2. Disponível em: http://proceedings.ciaiq.org/index.php/ciaiq2017/article/view/1219/1180 Acesso em 10/12/2017.

13. Bardin L. Análise de conteúdo. Reto LA, Pinheiro A, tradutores. Lisboa: Edições 70; 2010.

14. Saldaña J. The coding manual for qualitative researchers. London: Sage; 2013.

15. Vosgerau DSAR, Pocrifka DH, Simonian. M. Associação entre a técnica de análise de conteúdo e os ciclos de codificação: possibilidades a partir do software ATLAS. ti. RISTI. 2016;(19):93-106.

16. Moraes R. Análise de conteúdo. Educação. 1999;22(37):7-32.

17. Queiroz TLDA. Cavalcante PS. (2011). As contribuições do software Atlas Ti para a análise de relatos de experiência escritos. Anais do X Congresso Nacional de Educação, EDUCERE. Pontifica Universidade Católica do Paraná, Curitiba (2011). p. 11776-87.

18. Pocrifka DH. Inclusão digital nas políticas públicas para formação de professores em Pernambuco. (Dissertação de mestrado). Recife: Universidade Federal de Pernambuco; 2012.

19. Cubas JM. Acesso aos serviços de saúde mental por crianças e adolescentes com deficiência intelectual e/ou transtorno mental em situação de acolhimento. (Dissertação de Mestrado). Curitiba: Pontifícia Universidade Católica do Paraná; 2016.

20. Brasil. Ministério da Saúde. Lei n. 10.216, de 6 de abril de 2001. Dispõe sobre a proteção e os direitos das pessoas portadoras de transtornos mentais e redireciona o modelo assistencial em saúde mental. Diário Oficial da República Federativa do Brasil. Brasília. 2001.

21. Brasil. Ministério da Saúde. Portaria n 3.088, de 23 de dezembro de 2011. Institui a Rede de Atenção Psicossocial para pessoas com sofrimento ou transtorno mental e com necessidades decorrentes do uso de crack, álcool e outras drogas, no âmbito do Sistema Único de Saúde (SUS). Diário Oficial da República Federativa do Brasil, Brasília. 2011. 
22. Sandelowski, M. (2000). Combining Qualitative and Quantitative Sampling, Data Collection, and Analysis Techniques in Mixed-Method Studies Res. Nurs. Health. 2000; 23:246-55.

23. Günther H. Pesquisa Quantitativa: esta é a questão? Psic.: Teor. e Pesq. 2006;22(2):201-10.

24. Ferreira IRC, Vosgerau DSAR, Moysés SJ, Moysés ST Diplomas Normativos do Programa Saúde na Escola: análise de conteúdo associada à ferramenta ATLAS TI. Ciênc. saúde coletiva. 2012;17(12):3385-98.

25. Carvalho ILN, Gondin APS, Holanda TT, Alencar VP. Capsi: avanços e desafios após uma década de funcionamento. Cad. Bras. Saúde Mental. 2014;6(14):46-60.

26. Costello EJ, He J, Sampson NA, Kessler RC, Merikangas KR. Services for adolescent psychiatric disorders: 12-month data from the National Comorbidity Survey-Adolescent. Psychiatr Serv. 2014;65(3):359-66.

27. Couto MCV, Delgado PGG. Crianças e adolescentes na agenda política da saúde mental brasileira: inclusão tardia, desafios atuais. Psicol. Clin. 2015;27(1):17-40.

28. Patel V, Kieling C, Maulik PK, Divan G. Improving access to care for children with mental disorders: a global perspective. Arch Dis Child. 2013;98(5):323-7.

29. Becker AE, Kleinman A. Mental health and the global agenda. N Engl J Med. 2013;369:66-73.

30. Behrens D, Lear, JH, Price, OA. Improving access to children's mental health care: Lessons from a study of eleven states. A report prepared for The Center for Health and Health Care in Schools, George Washington University; 2013.

31. Salvador-Carulla, L, Martinez-Leal, R, Poole, M, Salinas-Perez, JA, Tamarit, J, Garcia-lbanez, J, et al. The mental health care gap in intellectual disabilities in Spain: impact analysis and knowledge-to-action plan. (J. Ment. Health Policy Econ. 2013;16(3): 131-41.

32. Surjus LTLS, Campos RTO. Deficiência intelectual e saúde mental: quando a fronteira vira território. Polis e Psique.2013;3(2):82-96.

33. Surjus LTLS. Campos RTO. Interface entre deficiência intelectual e saúde mental: revisão hermenêutica. Rev. Saúde Públ. 2014;18(3):532-40

34. Paim BR, Porta DD, Sarzi DM, Cardinal MF, Siqueira DF, Mello AL, Terra, et al. Atendimento ao adolescente usuário de substâncias psicoativas: papel do centro de atenção psicossocial. Cogitare enferm. 2017;22(1):1-7.

35. Gondim SMG, Bendassolli PF. The use of the qualitative content analysis in psychology: a critical review. Psicol. Estud. 2014;19(2):191-9.

36. McGorry P, Bates T, Birchwood M. Designing youth mental health services for the 21st century: examples from Australia, Ireland and the UK. Br J Psychiatry. 2013;202(54): 30-5.

37. Boydell KM, Hodgins M, Pignatiello A, Teshima J, Edwards H, Willis D. Using Technology to Deliver Mental Health Services to Children and Youth: A Scoping Review. J Can Acad Child Adolesc Psychiatry. 2014;23(2):87-99.

38. Taquette S. Análise de Dados de Pesquisa Qualitativa em Saúde. Anais do $5^{\circ}$ Congresso Ibero-Americano em Investigação Qualitativa (CIAIQ2016) e do 1st International Symposium on Qualitative Research (ISQR2016), 2016, Porto. v. 2 (2016): Atas - Investigação Qualitativa em Saúde. Porto. v. 2. p. 524-533. Disponível em: http:// http://proceedings.ciaiq.org/index.php/ciaiq2016/article/view/790. Acesso em 18/04/2017. 


\section{Endereço primeiro autor:}

João Mário Cubas

Pontifícia Universidade Católica do Paraná - PUCPR

Programa de Pós-Graduação em Tecnologia em Saúde - PPGTS

Rua Imaculada Conceição, 1155

Bairro: Prado Velho

CEP: 80215-901 - Curitiba - PR - Brasil

E-mail: joao.cubas@gmail.com

\section{Endereço para correspondência:}

Deborah Ribeiro Carvalho

Pontifícia Universidade Católica do Paraná - PUCPR

Programa de Pós-Graduação em Tecnologia em Saúde - PPGTS

Rua Imaculada Conceição, 1155

Bairro: Prado Velho

CEP: 80215-901 - Curitiba - PR - Brasil

E-mail: ribeiro.carvalho@pucpr.br 